\title{
Recurrent pneumonias and bronchiectasis associated a commom variable immunodeficiency
}

\author{
Thais Martins Souza \\ From 3rd WAO International Scientific Conference (WISC) 2014 \\ Rio de Janeiro, Brazil. 6-9 December 2014
}

\section{Introduction}

Commom variable immunodeficiency (CVI) is the second more comom cause of Immunodeficiency. Its prevalence is about 10000 a 50000 persons. Its incidente is about the first and second life's decade. Both genders are commited and it can be associated a several phenotypes such a recurrent inlfections,autoimmunity,enteropathy,neoplasms,polyclonal lymphocytic infiltration. The main recurrent infections are those that achieve lungs,paranasal sinuses and gastrointestinal . 60\% of the cases of CVI are associated a bronchiectasis. The diagnosis is based on current infections, reduction of IGG, igm and /or iga below 2 standarts desviations for age, age over 4 years old and excluding others causes of hypogammaglobulinemia.

\section{Objective}

The objective is to report the case of a patient who was diagnosed with CVI associated a recurrent pulmonary infections and bronchiectasis.

\section{Description of the case}

GOA,16 years old, 6 previous pneumonias. The first one was when she was 6 . She was hospitalized In two of those episodes and also showed weight loss .when she was 15 , the investigation was started. Sweat test and anti HIV were negative.low levels of Immunoglobulins were found (IgG= 20,1 mg/dl; IgM=32,2 mg/dl; IgA=7,2 mg/ $\mathrm{dl} ; \mathrm{IgE}=1,1 \mathrm{mg} / \mathrm{dl})$. The lymphocite immunophenotyping search was normal for her age $\left(C D 3=2939 \mathrm{~mm}^{3}\right.$, $\mathrm{CD} 4=693 \mathrm{~mm}^{3}, \mathrm{CD} 8=2011 \mathrm{~mm}^{3}, \mathrm{CD} 19=876 \mathrm{~mm}^{3}$ ). Tomografy of the chest presented suggestive areas of severe chronic bronchiectasis. After the exclusion of others hypogammaglobulinemias ' causes and the diagnostic of CVI being confirmed, the treatment was iniciated using intravenous imunoglobulin $400 \mathrm{mg} / \mathrm{kg} /$ day and antimicrobial prophylaxis with azitrhomicin. After initiation of treatment, the child didn $t$ have others infections and Gained satisfactory weight. Currently she is being monitorized In a immunology ambulatory.

\section{Conclusion}

We want to emphasize need to exclude CVID In patients that presents recurrent pneumonias, mainly when it's associated a bronchiectasis.

\section{Consent}

Written informed consent was obtained from the patient for publication of this abstract and any accompanying images. A copy of the written consent is available for review by the Editor of this journal.

Published: 8 April 2015

\section{References}

1. Conley ME, Notarangelo LD, Etzioni A: Diagnostic criteria for primary immunodeficiencies. Representing PAGID (Pan-American Group for Immunodeficiency) and ESID (European Society for Immunodeficiencies). Clinical immunology 1999, 93(3):190-7.

2. Chapel $\mathrm{H}$, Cunningham-Rundles $\mathrm{C}$ : Update in understanding common variable immunodeficiency disorders (CVIDs) and the management of patients with these conditions. British journal of haematology 2009, 145(6):709-27.

doi:10.1186/1939-4551-8-S1-A275

Cite this article as: Souza: Recurrent pneumonias and bronchiectasis associated a commom variable immunodeficiency. World Allergy Organization Journal 2015 8(Suppl 1):A275
Ufj, Brazil any medium, provided the original work is properly cited. The Creative Commons Public Domain Dedication waiver (http:// creativecommons.org/publicdomain/zero/1.0/) applies to the data made available in this article, unless otherwise stated. 